\title{
Caracterisation D'isolats Locaux De Metarhizium Spp, Champignon Entomopathogene De Cosmopolites Sordidus Germar, Isoles Des Bananeraies En Cote d'Ivoire
}

\section{Demby Laetitia Muriel Kouadio}

Doctorante, Institut National Polytechnique Houphouët Boigny,

Côte d'Ivoire

N'goran Aby

Attaché de recherche, Centre National de Recherche Agronomique,

Côte d'Ivoire

Kouabenan Abo

Maître de conférence, Institut National Polytechnique Houphouët Boigny,

Côte d'Ivoire

\section{Claude Zeze Kouadjo}

Attachée de recherche, Centre National de Recherche Agronomique,

Côte d'Ivoire

\section{Kouman Kobenan}

Directeur de recherche, Centre National de Recherche Agronomique,

Côte d'Ivoire

Siaka Traore

Chargé de recherche, Centre National de Recherche Agronomique,

Côte d'Ivoire

\section{Philippe Gnonhouri}

Ingénieur de recherche, Centre National de Recherche Agronomique,

Côte d'Ivoire

Doi: 10.19044/esj.2018.v14n21p73 URL:http://dx.doi.org/10.19044/esj.2018.v14n21p73

\begin{abstract}
Chemical insecticides have been commonly used against the black weevil, Cosmopolites sordidus, in banana plantations which caused huge economic and agricultural losses. Despite their benefits, pesticides can be hazardous to both humans and the environment. Intensive research in substituting chemical insecticides with biological control agents have been driven. The biological and molecular characteristics of ten strains of Metarhizium spp, isolated from dead weevils, collected in banana plantation in Côte d'Ivoire were studied. The study aims to identify the species of
\end{abstract}


Metarhizium sp. The biological and molecular characteristics namely: length and width of the spores, colour of aerial mycelium as well as its variation over time, colour on the setback of the box, production of the diffusible pigment and the analysis of the region ITS (Internal Transcribed Spacer) were evaluated. The colonies presented at first a flat white mycelium cottony or fluffy appearance on which the spores formed small islets. After 21 days of incubation at $32^{\circ} \mathrm{C}$. in the dark, the mycelium takes on the periphery a greenish coloration with a powdery appearance. The relief is fairly flat. The spores are ellipsoid in shape of $3 \mu \mathrm{m}$ in length and $1 \mu \mathrm{m}$. The amplification by ITS-DNA PCR generated a specific fragment of $550 \mathrm{bp}$. The analysis of the ITS region of the sequences of these ten (10) isolates showed maximal correspondence with different strains of M. anisopliae and Metarhizium pinghaense.

Keywords: Metarhizium, ITS, characteristics, Côte d'Ivoire

\section{Résumé}

L'utilisation de produits de synthèse contre le charançon noir, Cosmopolites sordidus, est très répandue dans les bananeraies pour réduire les pertes agricoles et économiques occasionnées. Les effets indésirables de ces produits impactent négativement la qualité de vie et menacent l'équilibre de l'environnement. Des alternatives à la lutte chimique parmi lesquelles, la lutte biologique sont ainsi envisagées. L'étude vise à identifier l'espèce de Metarhizium sp. : longueur et largeur des spores, couleur du mycélium aérien et sa variation au cours du temps, couleur du revers de la boite, production de pigment diffusible et l'analyse de la région ITS (Espaceur Interne Transcrit) ont été évalués. Les caractéristiques biologiques et moléculaires de dix souches de Metarhizium spp, isolées de charançons morts, collectés dans les aires de culture bananière en Côte d'Ivoire ont été étudiés. Les colonies ont présenté, au début, un mycélium blanc plat d'aspect cotonneux ou floconneux sur lequel les spores ont formé de petits ilots. Après 21 jours d'incubation à $32^{\circ} \mathrm{C}$ à l'obscurité, le mycélium prend à la périphérie une coloration verdâtre avec un aspect poudreux. Le relief est plat. Les spores sont de forme ellipsoïdale de $3 \mu \mathrm{m}$ de longueur et $1 \mu \mathrm{m}$ de large. L'amplification par ITSPCR de l'ADN a généré un fragment spécifique de 550 bp. L'analyse de la région ITS des séquences des dix (10) isolats, a montré une correspondance maximale avec différentes souches de $M$. anisopliae et $M$. pinghaense.

Mots clés : Metarhizium, ITS, caractéristiques, Côte d'Ivoire

\section{Introduction}

Les bananes et les plantains constituent une ressource alimentaire vitale pour plus de 400 millions d'habitants des zones intertropicales (Lassois 
et al., 2009). Elles concourent à la sécurité alimentaire, en raison de leur haute valeur énergétique (carbohydrates) et nutritionnelle (minéraux et vitamines notamment). Elles contribuent également au développement économique local et régional, en garantissant des revenus réguliers aux producteurs. Toutefois, leurs cultures sont soumises à des pressions parasitaires importantes dues à l'action des maladies et des insectes ravageurs, dont l'un des plus redoutables est le charançon noir du bananier (Gold et al., 2003).

Le charançon noir du bananier, Cosmopolites sordidus, occasionne d'énormes pertes économiques et agricoles dans les bananeraies. Les pertes de rendement dues aux attaques sont supérieures à $40 \%$ dans la plupart des cas. (Gold et al, 2002). Pour éliminer ce parasite, des insecticides de synthèse ont été utilisés comme solution appartenant aux familles : des organophosphorés (II), des pyréthrinoïdes (II), des phenylpyrazoles (II). La lutte chimique, avec pour conséquences l'accumulation de résidus de pesticides dans les bananes, est l'une des principales préoccupations de nombreux exploitants. Outre dans les bananes, des résidus dans les sols, dans les eaux, ... en somme, les effets non intentionnels de ces substances de synthèse ont conduit à des solutions alternatives dont la recherche d'agents de lutte biologique (Treverrow et al., 1993 ; Gold et al., 2003). Ainsi, des essais réalisés en Amérique, en Australie et en Afrique ont montré des résultats concluants avec les champignons entomopathogènes des genres Beauveria et Metarhizium (Nankinga, 1994 et 1997, Ochieng, 2001). Les champignons du genre Metarhizium testés comme biopesticide sont issus des espèces M. anisopliae, M. acridum, Metarhizium brunneum (anonyme, 2001, Diedhou et al., 2014, Rogge et al., 2017). En Côte d'Ivoire, Metarhizium sp., un champignon pathogène de Cosmopolites sordidus a été identifié sur les aires de culture bananière (Aby et al., 2009, Aby, 2013), en vue de la formulation d'un biopesticide pour la lutte contre ce redoutable ravageur. Les tests préliminaires (Aby, 2013) sur les isolats obtenus ont révélé des comportements variables selon les critères tels que : l'aptitude à provoquer la mycose mortelle chez l'hôte et la réaction aux différents pesticides courants en plantation. Ce résultat supposerait une relative diversité au sein de la population de Metarhizium en Côte d'Ivoire. A ce jour, dix isolats locaux de Metarhizium sp ont été identifiés sur les différentes aires de culture bananière en Côte d'Ivoire. La présente étude a pour objectif de caractériser ces isolats avant leur utilisation dans un programme de formulation de biopesticide.

\section{Matériel et methods Matériel}

Le matériel fongique est issu d'une collecte réalisée en 2008 dans différentes aires de production bananière des régions des Grands Ponts, de l'Agneby-tchassa et du Sud Comoé en Côte d'Ivoire. Il se compose de dix 
isolats de Metarhizium spp auxquels des noms de code ont été attribués selon la région de provenance. Les isolats : BAS6, BME2, et BME5 ont été récoltés dans la région des grands ponts, dans la plantation Batia. Deux autres isolats D23 et D24 sont issus de la plantation SAKJ Diby, dans la région Sud Comoé. Les isolats DME1, Elima A7 et METE sont issus de la plantation Elima, dans la région Sud Comoé. Les isolats EGL1, EGL2 sont issus respectivement des plantations d'Eglin Azaguié et d'Eglin Agboville.

\section{Méthodes}

\section{Caractérisation macroscopique des isolats Metarhizium sp.}

L'étude des caractéristiques macroscopiques des champignons entomopathogènes est largement utilisée pour caractériser le genre Metarhizium (Tulloch, 1976). La caractérisation macroscopique des isolats a été réalisée selon la méthode de Benserradj (2014). Des fragments mycéliens de $6 \mathrm{~mm}$ de diamètre ont été prélevés avec une anse au niveau du front de croissance et mis sur milieu de culture pomme de terre (Pomme de terre Dextrose Agar). L'incubation a eu lieu à l'obscurité à la température de $30^{\circ} \mathrm{C}$ $\pm 2^{\circ} \mathrm{C}$ pendant 21 jours. Des indices révélateurs sur l'identité des souches sont relevés à l'œil nu : couleur du mycélium aérien et sa variation au cours du temps, couleur du revers de la boite, production de pigment diffusible, présence ou absence de gouttelettes sur le revers de la boite, présence ou absence de gouttelettes sur le mycélium.

\section{Caractérisation microscopique et évaluation de la capacité de sporulation des isolats Metarhizium sp.}

Les observations ont porté sur les critères morphologiques de classification des espèces de Metarhizium sp. proposés par Fernandez et al. (2009), et Benserradj (2014). Pour ce faire, un fragment de thalle de chaque isolat est prélevé à l'aide d'une aiguille lancéolée et déposé sur une goutte d'eau distillée stérile entre lame et lamelle le tout monté sur un microscope optique pour observer les caractères microscopiques des organes. Il s'est agi notamment de la morphologie des spores (forme, longueur, largeur), des conidiophores et des phialides. En outre, un micromètre a servi à mesurer la longueur et la largeur des spores au grossissement x 40 .

L'évaluation de la capacité à sporuler a été réalisée par numération à l'aide d'un hématimètre de Malassez, selon la méthode décrite par Aby (2013). Pour cela, une suspension de spores est obtenue en raclant en surface de la culture fongique qui est mise ensuite dans un tube à essai contenant $100 \mathrm{ml}$ d'eau distillée. Une goutte de cette suspension de spores est déposée sur la lame de Malassez pour la numération. Quatre répétitions ont été réalisées par isolat. 


\section{Caractérisation moléculaire des isolats de Metarhizium sp.}

La caractérisation moléculaire des isolats de Metarhizium sp. s'est déroulée en quatre phases : l'extraction de l'ADN, l'amplification de la région ITS, l'analyse et la comparaison des séquences et enfin l'analyse phylogénétique. Les données collectées ont été analysées statistiquement.

Extraction de l'ADN : Les isolats de Metarhizium sp. ont été d'abord cultivés sur un milieu PDA, dans des boîtes de Pétri de $90 \mathrm{~mm}$ de diamètre. Après 21 jours d'incubation, à l'obscurité et maintenus à température ambiante, le mycélium de chaque isolat est récolté par grattage à l'aide d'une spatule stérile. L'extraction de l'ADN a été réalisée selon le procédé fourni par le kit d'extraction (Zymo Research, Etats Unis) de marque. La concentration de 1'ADN obtenu est mesurée au Nanodrop.

Amplification de l'Espaceur Intergenique Transcrit (ITS): La Réaction de Polymérisation en Chaîne (PCR) de la région ITS des extraits d'ADN a été effectuée en utilisant les amorces ITS1 (5'-TCCGTAGGTGAACCTGCGG3') et ITS4 (5'-TCCTCCGCTTATTGATATGC-3'), selon le processus décrit par Benserradj (2014). Le tampon de réaction $(50 \mu 1)$ de PCR, porté dans le thermocycleur, était composé de : $25 \mu \mathrm{L}$ One Taq Quick-Load $(2 \mathrm{X}), 1 \mu \mathrm{l}$ amorces ITS1 $(10 \mu \mathrm{M}), 1 \mu \mathrm{l}$ amorces ITS4 $(10 \mu \mathrm{M}), 10 \mu \mathrm{l}$ ADN, $13 \mu \mathrm{L}$ H20 PCR. Cette opération a été effectuée en plusieurs réactions de 45 cycles, avec une dénaturation initiale à $95^{\circ} \mathrm{C}$ pendant 10 minutes, suivie de 45 cycles de dénaturation à $95^{\circ} \mathrm{C}(10$ secondes $), 1^{\prime}$ hybridation à $58^{\circ} \mathrm{C}(10$ secondes $)$ et $1^{\prime}$ 'élongation à $72^{\circ} \mathrm{C}(40$ secondes $)$. Une élongation supplémentaire à $72^{\circ} \mathrm{C}(5$ minutes) a été réalisée. Un témoin négatif a été réalisé sans ADN. La révélation des résultats de la PCR a été faite par électrophorèse sur gel d'agarose à $1,2 \%$, préparée avec un tampon TBE $(0,045 \mathrm{M}$ Tris-borate et 0,001 M EDTA, PH8,0) à 70V, pendant une (1) heure. Le gel d'agarose a été précoloré avec du «SYBR Safe DNA ». Les tailles des fragments ont été mesurées à l'aide d'un marqueur de poids moléculaire de $100 \mathrm{pb}$ de type Invitrogen. Après migration, le gel a été observé par un système d'imagerie numérique sous lumière UV (Ultra Violet).

Analyse et comparaison des séquences : Tous les fragments amplifiés par PCR ont été séquencés avec la méthode Sanger. Les produits PCR ont été séquencés au laboratoire d'agroecologie de l'INRA (Institut National de la Recherche Agronomique) Toulouse. Les résultats du séquençage des souches fongiques ont été obtenus sous la forme d'électrophorégrammes bruts. Les séquences d'ADN obtenues ont été soumises à une recherche d'homologie de séquences en utilisant le programme BLAST (Basic local Alignement Search Tool) sur le site NCBI (National Center For Biotechnology Information) pour rechercher les séquences les plus proches déposées dans Genbank.

Après avoir aligné les séquences, les arbres phylogénétiques ont été construits avec le programme Mega version 7 (Kumar et al., 2008). La 
robustesse des arbres a été testée par la méthode du bootstrap (Felsentein, 1985).

Analyse des données : Les analyses statistiques ont été effectuées à l'aide du logiciel SPSS version 20. L'analyse de variance univariée au seuil de $5 \%$ a étudié la différence de sporulation observée.

\section{Résultats}

\section{Caractéristiques macroscopiques des isolats}

Les aspects culturaux ainsi que les caractères morphologiques chez Metarhizium issu des bananeraies ivoiriennes sont variables d'un isolat à un autre (figure 1 ; tableau 1).

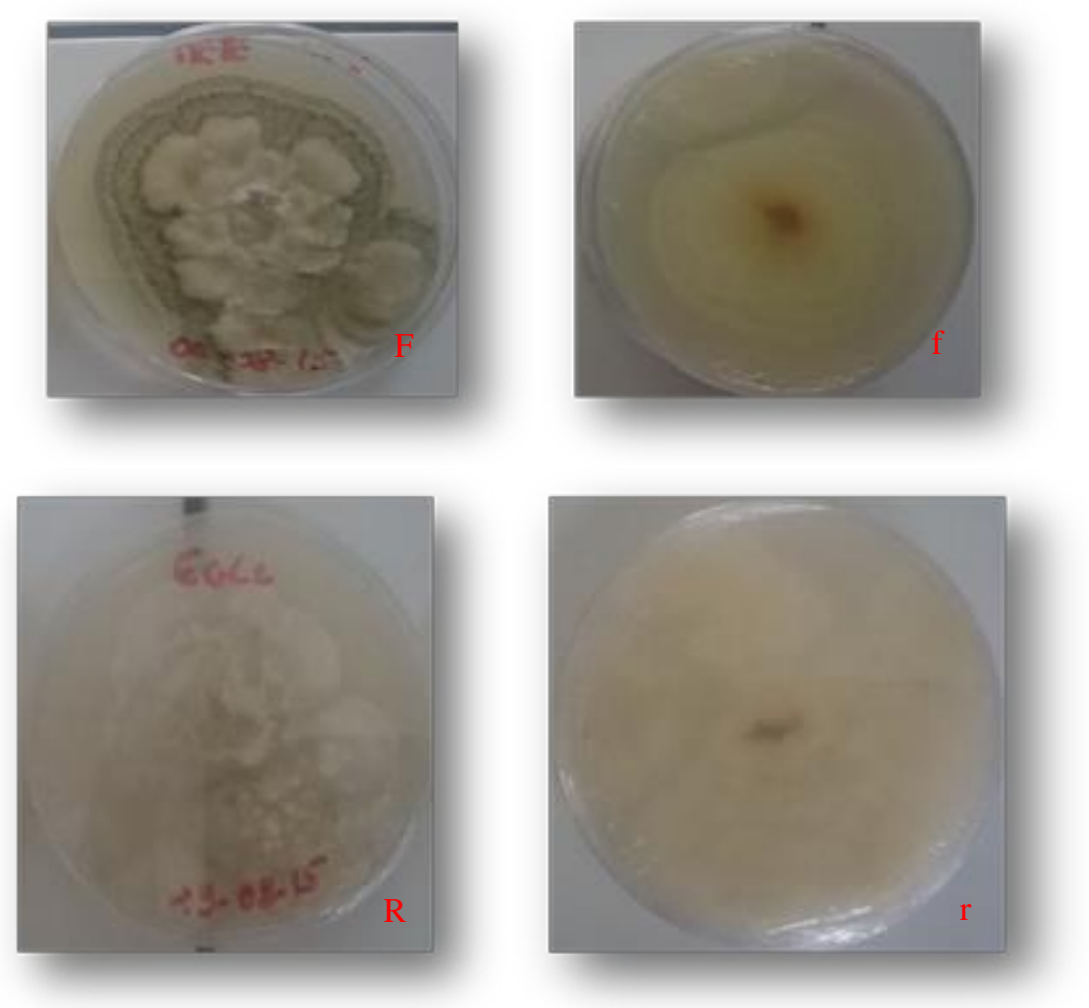

Figure 1: Isolats de Metarhizum sp. cultivés sur milieu PDA, F : isolat Mete vue de face , $\mathrm{f}$ : isolat Mete vue de revers, $\mathrm{R}$ : isolat Egl2 vue de face , $\mathrm{r}$ : isolat Eg12 vue de revers 
Tableau 1 : Caractéristiques macroscopiques d'isolats de Metarhizium $s p$

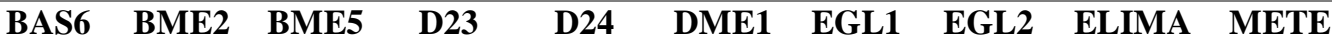
A7

\begin{tabular}{|c|c|c|c|c|c|c|c|c|c|c|}
\hline Relief & Plat & Plat & Plat & Plat & Plat & Plat & Plat & Plat & Plat & Plat \\
\hline Texture & $\mathrm{Ct} / \mathrm{Pr}$ & $\mathrm{Fl} / \mathrm{Pr}$ & $\mathrm{Fl} / \mathrm{Pr}$ & $\mathrm{Fl} / \mathrm{Pr}$ & $\mathrm{Fl} / \mathrm{Pr}$ & $\mathrm{Fl} / \mathrm{Pr}$ & $\mathrm{Ct} / \mathrm{Pr}$ & $\mathrm{Ct} / \mathrm{Pr}$ & $\mathrm{Ct} / \mathrm{Pr}$ & $\mathrm{Ct} / \mathrm{Pr}$ \\
\hline Aérobie & Aérien & Aérien & Aérien & Aérien & Aérien & Aérien & Aérien & Aérien & Aérien & Aérien \\
\hline Couleurs & $\begin{array}{c}\text { Bl } \\
\text { puis } \\
\text { vf }\end{array}$ & $\begin{array}{c}\text { Bl } \\
\text { puis vf }\end{array}$ & $\begin{array}{l}\text { Bl } \\
\text { puis vf }\end{array}$ & $\begin{array}{c}\text { B1 } \\
\text { puis } \\
\text { vf }\end{array}$ & $\begin{array}{l}\text { Bl puis } \\
\text { vf }\end{array}$ & $\begin{array}{l}\text { Bl } \\
\text { puis vf }\end{array}$ & $\mathrm{Bl}$ & $\begin{array}{c}\text { Bl } \\
\text { puis } \\
\text { vf }\end{array}$ & $\begin{array}{l}\text { Bl puis } \\
\text { vf }\end{array}$ & $\begin{array}{l}\text { Bl puis } \\
\text { vf }\end{array}$ \\
\hline Revers & $\mathrm{Bl}$ & Brun & Brun & $\mathrm{Bl}$ & Orangé & Brun & $\mathrm{Bl}$ & $\mathrm{Bl}$ & Orangé & Brun \\
\hline $\begin{array}{c}\text { Vitesse de } \\
\text { croissance }(\mathrm{mm} / \mathrm{j})\end{array}$ & 1 & 1 & 1 & 1 & 2 & 1 & 1 & 1 & 1 & 1 \\
\hline
\end{tabular}

Vf : vert foncé

$\mathrm{Bl}$ : blanc
$\mathrm{Br}:$ brun

Pr : poudreux
$\mathrm{Ct}$ : cotonneux

$\mathrm{Fl}$ : floconneux

Les colonies ont présenté, en début de croissance, un mycélium blanc plat d'aspect cotonneux pour EGL1 et EGL2 et Floconneux pour les isolats BAS6, BME2, BME5, D23, D24, DME1, ELIMA A7, et METE. Le mycélium présente, à la périphérie, une coloration verdâtre, avec un aspect poudreux formant des ilots.

Par contre, la souche EGL1 présente, jusqu'au 21ème jour, un mycélium avec une quasi absence de coloration verdâtre. Le relief est plat. Le revers est blanc ou jaune pour les isolats EGL1, EGL2, BAS6, D23, DME1 et brun orangé pour les isolats BME2, BME5, D24, ELIMA A7, METE.

Ces caractères culturaux ont permis de séparer les isolats en deux groupes. Les isolats Bas6, EGL1, EGL2, METE, et ELIMA A7 ont présenté une texture cotonneuse. Les isolats BME2, BME5, D23, D24, et DME1 ont quant à eux présentés une texture floconneuse.

\section{Caractéristiques microscopiques et potentiel de sporulation des isolats}

Le champignon présente, au microscope, un mycélium hyalin, cloisonné et ramifié. Les conidiophores sont aussi ramifiés. Les phialides ont une forme cylindrique ou légèrement gonflés sur le côté dans la zone d'insertion des spores. Les conidies de forme ellipsoïdale sont unicellulaires. Elles sont hyalines et ont une longueur moyenne de $3 \mu \mathrm{m}$ et une largeur moyenne $1 \mu \mathrm{m}$. Il n'y aucune différence significative entre les isolats, aussi bien pour la longueur que pour la largeur des conidies.

La capacité de sporulation des différents isolats est variable (figure 2). Les isolats ont présenté un potentiel de sporulation significativement différent (p0.05). Les isolats METE et D24 ont été les plus sporulants ; suivis des isolats BME5, DME1, EGL2 et ELIMA A7. Les autres isolats ont été faiblement 
sporulants. L’isolat EGL1 est celui qui présente la concentration en spores par millilitre la plus faible.

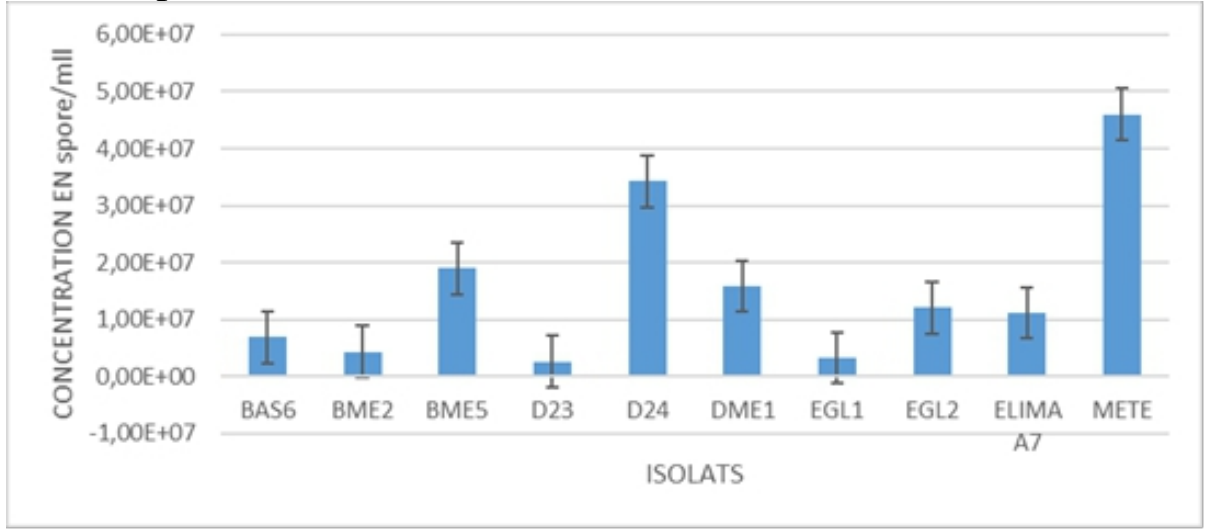

Figure 2 : Potentiel de sporulation des isolats locaux de Metarhizium sp

\section{Caractéristiques moléculaires des isolats de Metarhizium sp. :}

L'amplification des extraits d'ADN des 10 isolats de Metarhizium sp., avec la paire d'amorces universelles ITS1 et ITS4, a produit des fragments d'ADN dont la taille approximative est de 550 pb (figure 3 ).

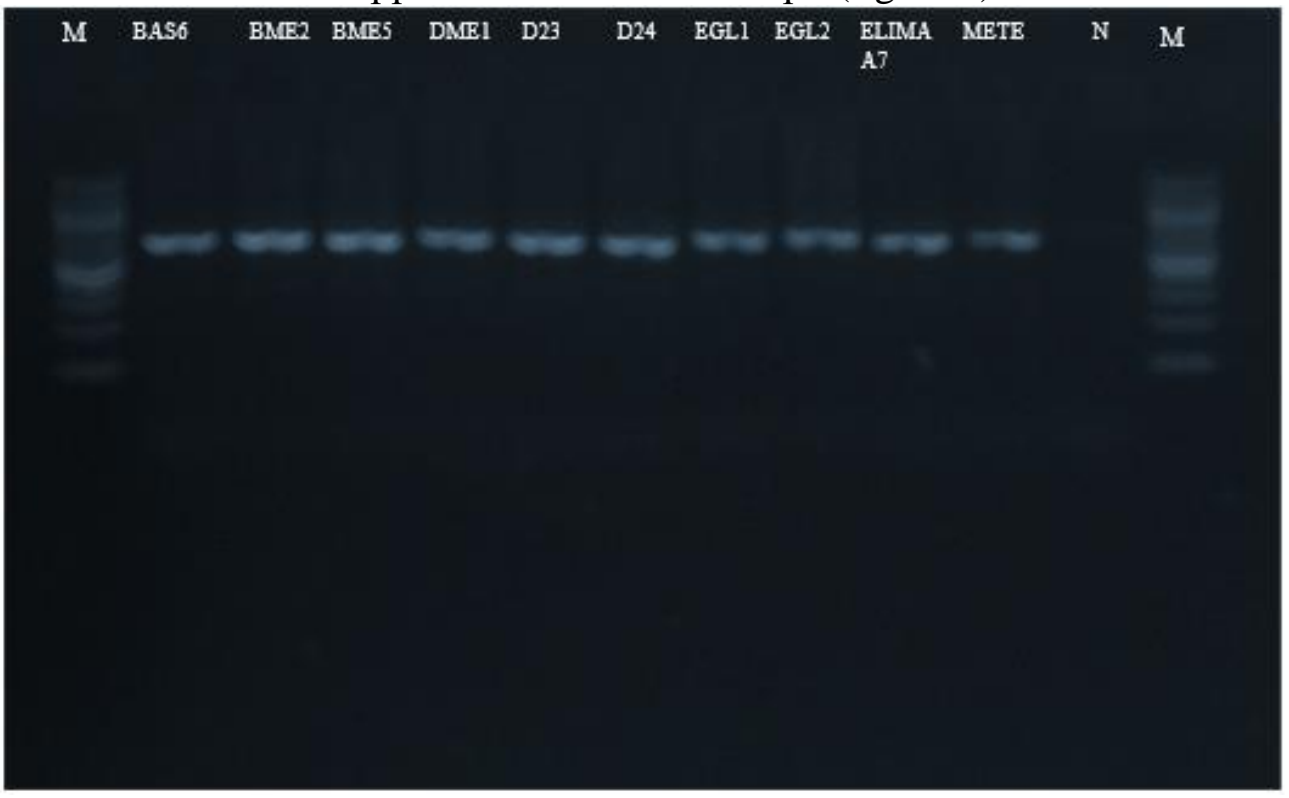

Figure 3 : Profil d'amplification de la région ITS par PCR de dix (10) isolats de Metarhizium sp collectés dans les bananeraies ivoiriennes en $2008 \mathrm{M}$ : marqueur, N : témoin négatif

Les produits de la PCR ont été séquencés et les séquences obtenues ont été comparées avec celles d'autres organismes de la base de données Genbank. L'analyse de la région ITS des dix (10) isolats, a montré une correspondance 
maximale avec différentes souches de $M$. anisopliae. Les séquences ont présenté une similarité de $98 \%$ à $100 \%$ avec les espèces : Metarhizium anisopliae strain Ma-62, Metarhizium anisopliae strain TK37, Metarhizium anisopliae strain Ma-64, Metarhizium anisopliae strain MAZL6 et Metarhizium anisopliae isolate KTU-32 (tableau 2). Seul l'isolat EGL 1 a obtenu comme deuxième espèce à correspondance maximale Metarhizium pinghaense strain GKVK, avec une correspondance de $99 \%$, sur l'ensemble des bases de la séquence de l'espèce.

Tableau 2 : Affiliation phylogénétique des dix isolats

\begin{tabular}{|c|c|c|c|}
\hline SOUCHES & IDENTITE & ESPECES PROCHES & $\begin{array}{l}\text { NUMERO } \\
\text { D'ACCESSION }\end{array}$ \\
\hline \multirow[t]{2}{*}{ BAS6 } & $100 \%$ & M. anisopliae strain Ma-62 & KU170588 \\
\hline & $100 \%$ & M. anisopliae strain MAZL6 & KF913493 \\
\hline \multirow[t]{2}{*}{ BME2 } & $99 \%$ & M. anisopliae strain Ma-62 & KU170588 \\
\hline & $99 \%$ & M. anisopliae strain MAZL6 & KF913493 \\
\hline \multirow[t]{2}{*}{ BME5 } & $100 \%$ & M. anisopliae strain MAZL6 & KF913493 \\
\hline & $99 \%$ & M. anisopliae strain Ma-62 & KU170588 \\
\hline \multirow[t]{2}{*}{ DME1 } & $99 \%$ & M. anisopliae strain MAZL6 & KF913493 \\
\hline & $99 \%$ & M. anisopliae strain Ma-62 & KU170588 \\
\hline \multirow[t]{2}{*}{ D23 } & $99 \%$ & M. anisopliae strain Ma-64 & KU170589 \\
\hline & $99 \%$ & Uncultured Metarhizium clone Ma-m1 & KP739826 \\
\hline \multirow[t]{2}{*}{ D24 } & $100 \%$ & M. anisopliae strain MAZL6 & KF913493 \\
\hline & $99 \%$ & M. anisopliae strain Ma-62 & KU170588 \\
\hline \multirow[t]{2}{*}{ EGL1 } & $99 \%$ & M. anisopliae isolate KTU-32 & FJ177479 \\
\hline & $99 \%$ & M. pinghaense strain GKVK & KM091861 \\
\hline \multirow[t]{2}{*}{ EGL2 } & $100 \%$ & M. anisopliae strain Ma-62 & KU170588 \\
\hline & $100 \%$ & M. anisopliae strain MAZL6 & KF913493 \\
\hline \multirow[t]{2}{*}{ ELIMA A7 } & $100 \%$ & M. anisopliae strain Ma-64 & KU170589 \\
\hline & $99 \%$ & Uncultured Metarhizium clone Ma-m1 & KP739826 \\
\hline \multirow[t]{2}{*}{ METE } & $99 \%$ & M. anisopliae strain TK37 & KU170589 \\
\hline & $99 \%$ & M. anisopliae strain Ma-62 & KP739826 \\
\hline
\end{tabular}

Les souches forment un clade homogène soutenu par une valeur bootstrap de 100. Cette homogénéité est perceptible au niveau des caractères macroscopiques où quelques différences sont observées. Un début de mutations de type allotypique est observé au niveau de l'isolat Egl1 (figure 4). 


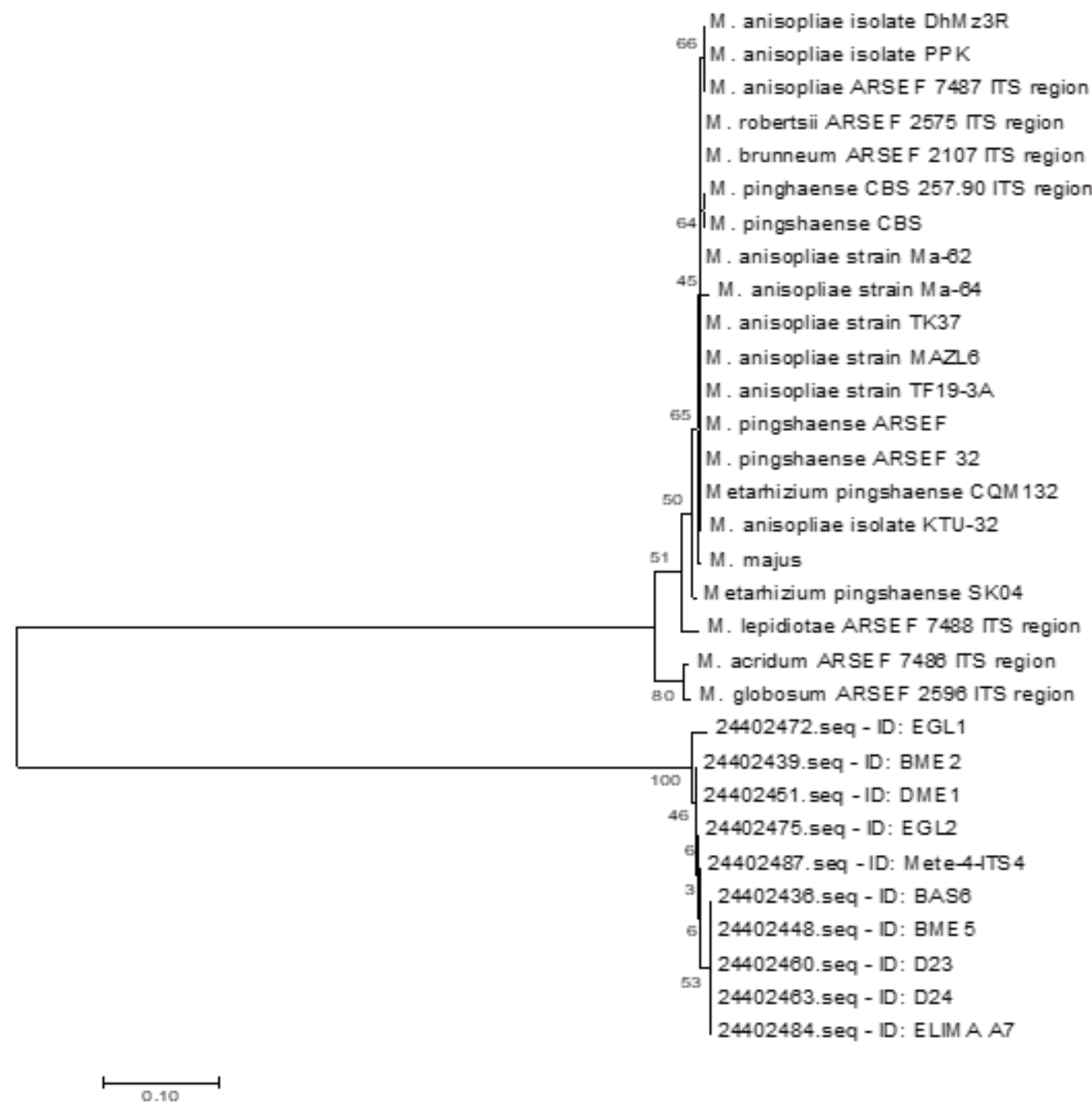

Figure 4 : Arbre phylogénétique selon la méthode des distances (Neighbor -Joining). Les distances évolutives ont été calculées en utilisant la méthode Kimura 2-parameter. Les valeurs mentionnées à l'origine des nœuds représentent le pourcentage après 500 bootstrap.

0.1 représente le nombre de substitution par position de nucléotide.

\section{Discussion}

Les dimensions des conidies des 10 isolats de Metarhizium spp. étudiés se rapprochent des dimensions de certaines souches de Metarhizium anisopliae. Toutefois, l'absence de différence entre les dimensions des conidies ne permet pas de les séparer sur la base de ce caractère. Ce constat a également réalisés par Bischoff et al. (2009) et Fernandes et al. (2010), qui démontrèrent l'impossibilité de ségréger les différentes espèces du genre Metarhizium, par les dimensions des conidies. 
Les données de la caractérisation macroscopique et microscopique ne permettent pas d'identifier clairement les isolats en présence. Entz et al. (2005) évoquèrent les difficultés d'identification des espèces en se basant sur des caractères morphologiques. Selon eux, exposées à des conditions environnementales et physiologiques différentes, les espèces pourraient présenter une morphologie différente. En conséquence l'examen morphologique n'est pas suffisant pour une identification précise des variétés et des espèces de Metarhizium spp.

Dans cette étude, l'analyse moléculaire a consisté à réaliser l'analyse des séquences de la région ITS, en utilisant les deux amorces (ITS1 : 5' TCC GTA GGT GAA CCT GCG G 3' et ITS4 : 5' TCC TCC GCT TAT TGA TAT GC 3') (Driver et al., 2000 ; Entz et al.,2005). Les données de séquençage confirment que neuf des dix souches étaient appariées à l'espèce $M$. anisopliae. La souche EGL1 était apparentée aux espèces $M$. anisopliae et $M$. pinghaense. La classification des espèces de Metarhizium s'est reposée historiquement sur les caractères morphologiques et biochimiques (Glare et al., 1996 ; Bridge et al., 1997). Cependant, la variation morphologique et le chevauchement des phénotypes chez certaines espèces rend cette classification difficile (Lomer et al., 2001 ; Glare et al., 1996) et complique l'utilisation des caractères morphologiques comme critères taxonomiques pour estimer la diversité interspécifique et intra spécifique des populations. Les méthodes moléculaires sont utilisées en complément des approches morphologiques car, par leur grande sensibilité, elles permettent la détection de variation entre les organismes à partir des séquences nucléotidiques (Dong et al., 2007, Entz et al., 2005, Rodriguez et al., 2004 ; Driver et al., 2000). Parmi les méthodologies employées, la réaction en chaîne de la polymérase (PCR) est largement pratiquée. La région ITS est l'outil généralement utilisée pour l'identification moléculaire des champignons (Driver et al., 2000, Benserradj, 2014). Elle est considérée comme conservée au niveau intra-spécifique et variable entre espèces. Il représente donc un bon marqueur pour la détection des espèces de champignons.L'amplification a donné un fragment unique de $550 \mathrm{pb}$ pour tous les isolats. De Stefano et al. (2004) ont analysé la même région et ont obtenu un fragment de $540 \mathrm{pb}$ pour M. anisopliae var. anisopliae souche E9, B / Vi et C isolé au Brésil et 600 pb pour M. anisopliae souche 14 isolé en Australie. Plusieurs auteurs ont accepté de considérer l'ITS comme étant le premier choix pour établir le code bar $\mathrm{ADN}$ du règne des champignons. La région ITS est fréquemment utilisée dans l'analyse phylogénétique des champignons entomopathogènes (Nikoh et Fukatsu, 2000). Les produits ont été séquencés et comparés aux séquences contenues dans la Genbank en utilisant le BLASTN. Ils ont montré une identité de $98 \%$ à $100 \%$, avec des variétés de $M$. anisopliae. L'analyse phylogénétique a été réalisée selon la méthode des distances (Neighbor -Joining) à l'aide du logiciel Mega 7.0.1.4. Le 
dendrogramme a été regroupé en 4 classes. Les dix isolats ont formé un groupe monophylétique, soutenu par une valeur bootstrap de $99 \%$. Ce groupe monophylétique montre la différence entre les séquences des dix souches et celles enregistrées dans la Genbank. Les faibles valeurs des branches entre les différentes souches mettent en exergue les limites de l'étude de la région ITS pour la différenciation de séquences voisines. Cette observation a été également réalisée par Bischoff et al. (2009), Bruns (2001) et Geiser (2004), qui montrèrent la difficulté à partir des séquences de la région ITS à différencier des séquences voisines. Les travaux de Bischoff et al. (2009) conseillèrent l'utilisation d'une PCR multiplex couvrant les régions EF-1 $\alpha$, $\mathrm{RPB} 1, \mathrm{RPB} 2$ et du gène $\beta$-tubulin pour différencier les espèces du genre de Metarhizium.

\section{Conclusion}

$\mathrm{Au}$ terme de cette étude, il ressort, que l'évaluation des caractères morphologiques est inappropriée pour ségréger les différents isolats, les isolats ayant présentés des caractères communs sur de nombreux critères. Seul l'isolat EGL1 a présenté un caractère morphologique singulier. De nombreux chevauchements de ces caractères morphologiques peuvent conduire à des erreurs d'identification ou à des identifications imprécises. Les analyses moléculaires ont permis de confirmer les caractères phénotypiques observés. Les isolats BAS6, BME2, BME5, DME1, D23, D24, EGL2, ELIMA A7, et METE peuvent être affiliées à l'espèce $M$. anisopliae. Le caractère phénotypique singulier de l'isolat EGL1 a été confirmé par l'analyse de la séquence nucléotidique de la région ITS 4 de cette souche. En effet, elle a présenté comme espèce à deuxième correspondance maximale une variété de Metarhizium pingshaense. Tant au niveau macroscopique que génétique, il présente une singularité vis-à-vis des neuf autres isolats. C'est un début de spéciation qui est ainsi observé. L'analyse phylogénétique des neuf (9) autres isolats n'a pu, tout comme l'analyse phénotypique, établir une différence distincte entre les différentes isolats. L'utilisation d'autres marqueurs pourront nous donner de plus amples informations sur la diversité existant entre les souches de $M$. anisopliae de cette étude. Une PCR multiplex couvrant les régions EF-1 $\alpha, \mathrm{RPB} 1, \mathrm{RPB} 2$ et du gène $\beta$-tubulin permettra d'étudier la diversité variétale au sein des différentes souches.

Remerciements : Nous tenons à remercier le FIRCA (Fond Interprofessionnel pour la Recherche et le Conseil Agricole) pour 1'appui financier.

\section{References:}

1. Aby, N, Kehe, M, Kobenan K.\& Gnonhouri P (2008). Recherche de souches locales de champignons parasites des charançons du bananier 
dans les principaux bassins de production de banane dessert en Côte d'Ivoire, Avril-Septembre 2008. Premier rapport d'étape Projet FIRCA/CNRA, Décembre 2008. 22p.

2. Aby, N. (2013). Lutte biologique contre le charançon noir du bananier Cosmopolites sordidus Germar (Coléoptère, Curculinodae) en Côte d'Ivoire : Caractéristiques entomopthologiques d'isolats locaux de Metarhizium sp. sur les populations au laboratoire et en bananeraie, mémoire de thèse de Doctorat présentée à l'UFR biosciences de l'université de Cocody, p169,

3. Anonyme1(2003).https://www3.epa.gov/pesticides/chem_search/reg_ actions/registration/fs_PC-029056_01-Jun-03.pdf

4. Bridge, P. D., Prior, C., Sagbohan, J., Lomer C. J., Carey, M., \& Buddie, A. (1997). Molecular characterization of isolates of Metarhizium from locusts and grasshoppers. Biodiversity and Conservation 6: 177-189.

5. Bruns, T. D. (2001),. ITS Reality. Inoculum. 52: 2-3

6. Entz, C., Johnson, L., \& Kawchuk, L. (2005). Development of a PCRbased diagnostic assay for the specific detection of the entomopathogenic fungus Metarhizium anisopliae var.acridum, Mycol. Res.109(11) : 1302-1312

7. Fernandes, É.K.K., Keyser, C.A., Chong, J.P., Rangel, D.E.N., Miller, M.P., \& Roberts, D.W (2010). Characterization of Metarhizium species and varieties based on molecular analysis, heat tolerance and cold activity, Journal of applied microbiology Volume 108, Issue1, Pages 115-128

8. Geiser, D.M., Jimenez-Gasco, M.D., Kang, S.C., Makalowska, I., Veeraraghavan, N., Ward, T.J., Zhang, N., Kuldau, G.A., \& O’Donnell, K. ( 2004). Fusarium-id v. 1.0: a dna sequence database for identifying Fusarium. European Journal of Plant Pathology 110:473-479

9. Glare, T. R., Milner, R. J., \& Beaton, C. D. (1996). Variation in Metarhizium, a genus of fungal pathogens attacking Orthoptera:is phialide morphology a useful criterion Journal of Orthoptera Research5: 19-27.

10. Gold C.S. \& Tushemereiwe W., 2002 : Aperçu de la recherche sur le charançon du bananier en Ouganda. Promusa $n^{\circ} 9,10 \mathrm{p}$.

11. Gold, C.S., \& Messiaen, S. (2000). le charançon du bananier Cosmopolites sordidus, Parasites et ravageurs des Musa: fiche technique $\mathrm{n}^{\circ} 4$, Réseau international pour l'amélioration de la banane et de la banane plantain, Parc Scientifique Agropolis II. 
12. Lassois, L., Busogoro, J. P., \& Jijakli, H. (2009). La banane : de son origine à sa commercialisation. Biotechnology, Agronomy, Society and Environment13, 575-586.

13. Lomer, C. J., Bateman, R. P., Johnson, D. L., Langewald, J., \& Thomas, M. (2001). Biological control of locusts and grasshoppers. Annual Review of Entomology 46: 667-702.

14. Nikoh, N., \& Fukatsu, T. (2000). Interkingdom host jumping underground: phylogenetic analysis of entomoparasitic fungi of the genus Cordyceps. Molecular Biology and Evolution 17,629-638.

15. Rogge, S. A., Mayerhofer, J., Enkerli, J., Bacher, S., \& Grabenweger, G. (2017). Preventive application of an entomopathogenic fungus in cover crops for wireworm control,BioControl. 